\title{
Fecundación in vitro postmortem
}

\author{
In vitro fertilizationpostmortem \\ Postmortem fertilização in vitro
}

\author{
Patricia Sánchez Ruiz ${ }^{1}$, Nerea Martínez Castellón², Eloisa Fernández Ordóñez \\ ${ }^{1}$ Enfermera especialista en obstetricia y ginecología. Hospital Comarcal de la Axarquía. Vélez Málaga (Málaga). \\ ${ }^{2}$ Enfermera especialista en obstetricia y ginecología. Distrito Sanitario Málaga-Guadalhorce. C.S Coín. \\ ${ }^{3}$ Enfermera especialista en obstetricia y ginecología. Hospital Universitario Virgen de la Victoria (Málaga).
}

Cómo citar este artículo en edición digital: Sanchez-Ruiz, P., Martinez-Castellon, N., \& Fernandez-Ordoñez, E. (2018).

Fecundación in vitro postmortem. Cultura de los Cuidados (Edición digital), 22(50).

Recuperado de http://dx.doi.org/10.14198/cuid.2018.50.16

Correspondencia: Patricia Sánchez Ruiz. C/ Aristófanes, 18, bloque 4, 2², CP 29010, Málaga. Tlf 652073397

Correo electrónico: patix1986@hotmail.com

Recibido: 08/06/2017; Aceptado: 13/10/2017

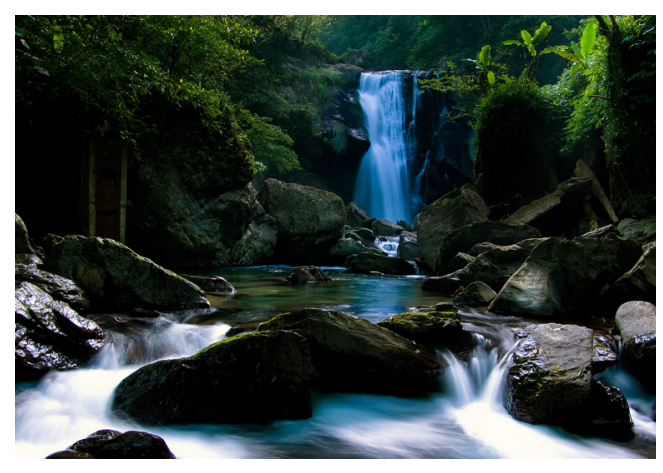

\section{ABSTRACT}

Postmortem IVF is a technique by semen is extracted from the deceased man to fertilize the egg of his wife. The objective of this review of the literature is to know and compare the different options of postmortem IVF in several countries. Methodology: literature review in different databases that return a search in English or Spanish with high relevance with the subject to be treated and available in full text as inclusion criteria, being the absence of any of the above exclusion criteria. Outcome: Currently in Spain it is allowed to perform this technique with a number of requirements and under legal conditions, however in other countries there is a lot of discrepancy, some are not allowed to use this technique under any concept, while in others access She is free for every widow woman who wants it within the first hours of the man's death. Discussion: Few countries have a current legislation on postmortem IVF, and very few countries allow the technique to be performed, even with the authorization of the male prior to death. Conclusions: There is a lot of controversy about the legislation in the different countries, so a deeper investigation on this topic would be necessary.

Keywords: Postmortem IVF, assisted reproduction, predeceasing, legislation

\section{RESUMEN}

La fecundación in vitro postmortem (FIV postmortem) es una técnica mediante la cual se extrae semen del hombre ya fallecido para fecundar el óvulo de su esposa viva. El objetivo de esta revisión de la literatura es conocer y comparar las distintas opciones de la FIV postmortem en varios países. Metodología: revisión de la literatura en diferentes bases de datos que nos devuelva una búsqueda en inglés o español con alta relevancia con el tema a tratar y disponible en texto completo como criterios de inclusión. Resultado: Actualmente en España se permite realizar esta técnica con una serie de requisitos y bajo unas condiciones legales, sin embargo existen muchas diferen- 
cias entre distintos países, en algunos no se permite el uso de esta técnica bajo ningún concepto, mientras que en otros el acceso a ella es libre para toda mujer viuda que lo desee dentro de las primeras horas del fallecimiento del hombre. Discusión: aún son pocos los países que tienen una legislación específica en cuanto a la FIV postmortem, y muy pocos los que permiten realizar la técnica, incluso teniendo la autorización del varón previa al fallecimiento. Conclusiones: Existe gran controversia en cuanto a la legislación en los diferentes países, por lo que sería necesario una investigación más profunda sobre este tema.

Palabras Clave: FIV postmortem, legislación, reproducción asistida, premoriencia

\section{RESUMO}

IVF postmortem é uma técnica pela qual o sêmen é extraído do homem morto agora para fertilizar o óvulo da esposa viva. O objetivo desta revisão de literatura é determinar e comparar as diferentes opções de IVF postmortem em vários países. Metodologia: revisão da literatura nas bases de dados diferentes que você retornar uma pesquisa em Inglês ou Espanhol com alta relevância para o tema e está disponível em texto completo como critério de inclusão, com a ausência de qualquer um dos critérios de exclusão acima. Resultado: atualmente na Espanha estão autorizados a realizar esta técnica com uma série de requisitos e condições legais, mas em outros países há muito discrepância em algum uso desta técnica de qualquer forma não são permitidos, enquanto no acesso outros é livre para qualquer viúva que deseja dentro das primeiras horas da morte do homem. Discussão: Poucos países têm legislação sobre postmortem fertilização in vitro, e muito poucos que permitem a técnica, mesmo com autorização prévia do sexo masculino até a morte. Conclusões: Há muita controvérsia sobre a legislação em diferentes países, de modo mais pesquisas sobre este assunto seria necessário.

Palavras chave: IVF postmortem, reprodução assistida, predeceasing, legislação.

\section{INTRODUCCIÓN}

La fecundación in vitro (FIV) es una técnica de reproducción médica asistida que surgió a finales de los setenta. La técnica consiste en la hiperestimulación ovárica de la mujer, con posterior extracción del ovocito y fecundación (con semen de donante o del marido) en el laboratorio para su posterior transferencia al útero materno de nuevo. En el año 1978 en Cambridge nació por primera vez un bebé cuya gestación fue conseguida por FIV. La técnica FIV se implantó plenamente en España en la década de los ochenta (Escudero Velando, LE, 2012). Cuando hablamos de fecundación post-mortem nos estamos refiriendo a realizar una fecundación in vitro con el semen de un hombre fallecido, lo cual puede causar conflictos éticos y legales en la familia y en la sociedad. La técnica en sí puede resultar de dos maneras, o bien que el semen ya haya sido extraído y esté congelado, para lo cual el hombre tuvo que dar su consentimiento en vida, o bien que la mujer haga extraer el semen del hombre ya fallecido. Otra variante es la transferencia de embriones, en la cual el óvulo ya estuviera fecundado y congelado listo para ser implantado en un tratamiento de reproducción asistida y es usado una vez el hombre ha fallecido, aunque este uso suele ocurrir cuando el varón fallece de forma repentina.

La extracción y posterior almacenamiento para un futuro uso suele darse en casos en los que el hombre es diagnosticado de una enfer- 
medad terminal o en la que a causa del tratamiento, su material genético pueda quedar destrozado, impidiendo así la oportunidad de ser padre en un futuro. En estos casos, lo más probable es que el varón haya dejado constancia de su deseo de ser padre o bien a través de un consentimiento informado, escritura pública o testamento vital en el momento de la criopreservación, por lo tanto no debe haber problema legal en realizar la fecundación post mortem.

La primera extracción de semen post mortem con fines reproductivos de la que se tiene constancia ocurrió en 1980, y seguidamente fueron surgiendo más casos, aunque han creado polémica y algunos países se han visto obligados a crear una ley en la que basarse a la hora de dar los permisos pertinentes para proceder a la extracción y utilización con fines reproductivos del semen. Este tema suscita mucha polémica, ya que de esta técnica nacerá un niño huérfano de padre. El hecho de extraer el semen del marido ya fallecido y fecundar a la mujer no significa que se vaya a producir un embarazo, tal y como se muestra en el estudio de Tash (2003), en el cual de 22 familias que lo solicitaron solo 4 eran candidatas y no se produjo ningún embarazo.

Existen varias formas de hacerse con el material genético del varón (Gómez Jerez, P.L, 2006):

1- Que estuviese criopreservado desde antes del fallecimiento (en cuyo caso es de esperar que tenga firmado un consentimiento informado para tratar la muestra).

2- Por biopsia cadavérica del testículo (es necesario el consentimiento previo para realizar la ablación de los testículos).

3- Por flushing vaginal (el semen eyaculado en un acto sexual no puede ser susceptible de apropiación).
La ley española permite el uso de esta técnica siempre y cuando el hombre hubiese dado consentimiento por escrito o en el testamento antes de fallecer y la fecundación sea realizada en los primeros 6 meses tras el fallecimiento del marido. El bebé tendrá derecho a reconocimiento filial y por tanto será heredero del fallecido. Antiguamente la ley española 35/1988, en su artículo 9.1 sobre técnicas de reproducción asistida, reconoce que la muerte del varón es un impedimento legal para la utilización de su material reproductor por la mujer sometida a la aplicación de las técnicas y sólo en caso de que la pareja estuviera sometiéndose a un tratamiento de reproducción asistida en el momento del fallecimiento del marido, sería posible una transferencia de preembriones en el plazo máximo de 6 meses desde el momento del fallecimiento (Ley 35, 1988). Tras la modificación y posterior derogación de este código surge la actual ley de 2006 (Ley 14, 2006. Sobre técnicas de reproducción humana asistida. 26 de mayo. BOE), con la cual se regularizó la situación en España (Ley 14, 2006).

\section{Objetivos:}

- Conocer la legislación actual en España sobre fecundación postmortem

- Comparar la legislación actual española con las leyes de otros países en cuanto a materia de fecundación post mortem.

\section{METODOLOGÍA}

La metodología utilizada para la realización del presente estudio consiste en una búsqueda de la literatura existente sobre el tema a tratar, mediante la cual hemos obtenido distintos artículos y accesos a páginas web de interés para realizar el presente trabajo de tipo divulgativo. Se han utilizado diversas palabras clave como son fecundación in vitro postmortem, 
legislación, premoriencia, reproducción asistida. Todos ellos combinando el idioma inglés con español y con los descriptores booleanos AND y OR.

El motor de búsqueda de la información han sido el buscador google, para tener acceso a la actual legislación española sobre el tema a tratar y buscadores de bases de datos de salud, como puede ser google académico y distintas bases de datos de salud como Cochrane Plus, WOS y Pubmed, en las cuales hemos introducido las palabras clave con distintas combinaciones. La base de datos Cochrane no nos ha devuelto ningún artículo relevante para incluir en este trabajo divulgativo. Google académico, con la combinación anterior de palabras clave, nos ha devuelto 1820 resultados (sin incluir citas ni patentes), de los cuales hemos rescatado 7 por su relevancia con el tema y por disponer del texto completo. La base de datos Pubmed nos ha devuelto 44 referencias, de las cuales sólo uno tiene relevancia con nuestro estudio, pero no pudimos incluir por no tener acceso al texto completo.

Por su lado, Web of Science nos ha devuelto 68 resultados, de los cuales hemos incluido en este texto 3.m Los criterios de inclusión han sido: texto con importante relevancia con respecto al trabajo que pretendemos divulgar, idioma español o inglés, acceso al texto completo.

\section{DESARROLLO DEL TEMA}

\section{Situación actual en España}

Actualmente es posible la fecundación in vitro post-mortem siempre y cuando el varón ya fallecido haya dejado constancia por escrito (bien en un consentimiento, una escritura pública o un testamento) de su deseo de ser padre en caso de fallecimiento en los 12 meses siguientes al éxitus (el plazo ha sido ampliado el doble de lo que estipulaba la anterior ley) (Pérez Gallardo, 2007). Si el material genético resulta de un tratamiento al que se hubiere sometido previamente el fallecido para la transferencia de preembriones, el hijo nacido podrá llevar los apellidos del padre y tendrá derecho legal en cuanto a filiación (Ley 14, 2006).

Si por el contrario, el material genético no resultase de un tratamiento previo, el hijo nacido no tendrá derecho legal a la filiación ni relación jurídica con el marido fallecido (Ley 14, 2006).

Esta norma puede parecer incoherente con el artículo 39 de la Constitución española de 1978 (Constitución Española, 1978) en la que se cita textualmente "Los padres deben prestar asistencia de todo orden a los hijos habidos dentro o fuera del matrimonio, durante su minoría de edad y en los demás casos en que legalmente proceda.", ya que en la actual ley sobre técnicas de reproducción humana asistida, el artículo que trata el caso de la premoriencia del marido, da por hecho que ese futuro hijo nacerá huérfano de padre, en referencia a esta contradicción, hay autores que opinan que la Ley 14/2006 es anticonstitucional por vulnerar el derecho fundamental de los niños de crecer con padre y madre y el derecho a conocer a ambos progenitores, lo cual cita el artículo 39.3 de la Constitución española de 1978 (Rodríguez Guitián, 2015).

Guitián (2015) refiere que existen varios casos en los que el hijo no podrá conocer al padre, por ejemplo si la madre se niega a desvelar con quién mantuvo relaciones sexuales, tras una violación, y otro caso es el de la fecundación postmortem, en el cual el hecho de que el niño carezca de padre es evitable $y$ previsible, y que el niño tiene derecho a conocer a ambos progenitores según el artículo 7 de la Convención de los Derechos del Niño de 
20 de noviembre de 1989 (Rodríguez Guitián, 2015).

En el caso de la transferencia de embriones post mortem plantea otro supuesto distinto, ya que, para empezar, la legislación lo trata en otro artículo diferente, el tema del consentimiento no es necesario (ya que se sobreentiende que si el óvulo ya ha sido fecundado es porque deseaba ser padre junto con su mujer), además en estos casos el consentimiento suele estar firmado previo al fallecimiento, y en este consentimiento, en el cual el hombre indica su autorización a utilizar su material genético con fines reproductivos se sobreentiende su deseo de ser padre (Ley 14, 2006) y los tiempos de realización de la técnica son diferentes (en concreto no se indica tiempo). En este caso estaríamos ante un hijo póstumo, ya que la fecundación se produjo con el padre en vida, pero sólo podría hacerse la transferencia de embriones en caso de que se hubieran fecundado mientras el padre estaba vivo, esto quiere decir, que si el padre fallece y el material aún no ha sido usado, sino que permanece criocongelado, no sería posible la transferencia preembrionaria, y además en ese caso la legislación española no contempla la filiación tendría que haber un consentimiento específico en el cual el marido indicara con claridad su deseo de ser padre incluso si falleciera. (Pastrana Sánchez, 2014),

Es necesario velar por los intereses de todos los implicado: en el caso de la mujer, no quedaría privada de su derecho a ser madre, ya que podría tener hijos con otra pareja o bien podría someterse a un tratamiento de reproducción médica asistida con un donante anónimo. Por este motivo, el hecho de realizar una fecundación in vitro con el semen de un cadáver y traer al mundo a un niño cuyo padre falleció meses antes de su concepción podría ser interpretado por algunas personas como un acto de egoísmo.

Se describen varias razones para rechazar la fecundación in vitro post mortem, según Gómez Pérez (2006), entre ellas están :

1. El derecho al hijo y los derechos y el interés del hijo. El ser humano como fin en sí mismo y sujeto de derechos.

2. Privación del derecho a tener un padre.

3. Procreación responsable.

4. La motivación de la paternidad post mortem es contraria al interés del hijo.

5. Desviación de la finalidad terapéutica de las técnicas.

6. El Estado tiene la responsabilidad de asegurar que el hijo nacido de las técnicas de procreación asistida goce de las condiciones óptimas para la for m a ción y desarrollo de su personalidad.

\section{Problemas sucesorios.}

Todo ello suscita un dilema ético y moral en el cual deben primar los beneficios hacia el futuro hijo nonato, ya que, como se ha mencionado anteriormente, la madre no queda privada del hecho y disfrute de la maternidad.

\section{Situación Europea}

El ordenamiento Italiano (40/2004127) prohíbe expresamente la fecundación post mortem, de hecho, la reproducción asistida no es una alternativa para tener un hijo a menos que sea en último recurso y solo en el caso de parejas heterosexuales, mayores de edad y en edad potencialmente fértil y unidos en matrimonio o pareja de hecho. Tampoco está permitida la transferencia de preembriones fecundados antes del fallecimiento del padre si tal transferencia se produce después del falle- 
cimiento de éste. (Rodríguez Guitián, 2015), (Pastrana Sánchez, 2014).

En Francia tampoco está permitido, recogiéndose en el Código Civil francés, en el título VII, Sección IV. El artículo L. 2141-2 del Código francés de Salud Pública, que regula la reproducción asistida. En este código se señala, al igual que en el italiano, que para poder acceder al tratamiento médico es necesario estar unidos en vínculo o ser pareja estable, en edad de procrear y "vivos", por lo que no se contempla la fecundación postmortem en ninguna de sus modalidades. (Rodríguez Guitián, 2015), (Pastrana Sánchez, 2014).

Alemania castiga con pena de cárcel a quien fecunde un óvulo con el semen de un fallecido, esto consta en la Embryonenschutzgesetzvom. I 3. Dezember 1990, no castigándose a la mujer por tal hecho, sólo al personal sanitario (o no sanitario) implicado en el proceso. Sin embargo, la mujer sí podrá gestar los embriones si estos han sido fecundados previa la muerte del marido, por lo que en Alemania sí está permitida la transferencia de embriones aunque el marido haya fallecido (Rodríguez Guitián, 2015).

La legislación sueca también prohíbe la fecundación postmortem (Vega, Vega, \& Martinez Baza, 2004).

Reino Unido sí cuenta con legislación relativa a este tema (la cual entró en vigor en el año 2009). Para poder proceder a la inseminación postmortem debe existir un consentimiento previo por parte del hombre fallecido. El semen deberá extraerse dentro de las primeras 24 horas del fallecimiento y la FIV o inseminación o transferencia de embriones deberá producirse dentro de los 42 días posteriores al fallecimiento. No habrá problemas para inscribir al bebé como hijo del fallecido en el registro, pero se debe tener en cuenta que el fallecido constará como padre de modo simbólico, no habiendo repercusión legal en dicho acto (Rodríguez Guitián, 2015) (Muñoz de la Fuente, ,2010).

En Holanda la legislación es similar a la de Reino Unido con la excepción de que sí habrá derechos jurídicos, pudiendo llevar el hijo los apellidos del padre y constando en los registros para la nacionalidad y los derechos hereditarios (Rodríguez Guitián, 2015).

Portugal prohíbe expresamente la FIV o inseminación con semen del marido fallecido, incluso aunque éste haya dado su consentimiento por escrito con anterioridad al desenlace, sin embargo sí está autorizada la transferencia preembrionaria siempre y cuando se haya realizado mientras el marido o compañero permanecía con vida (Rodríguez Guitián, 2015).

La legislación en Bélgica es bastante permisiva en cuando a la reproducción asistida ya que, por ejemplo, fue el primer país es consentir la libre elección del sexo. En este país sí está permitida la fecundación postmortem incluso sin consentimiento previo escrito, con la única salvedad de un límite temporal que, no podrá ser inferior a 6 meses ni superior a 2 años $(\mathrm{Mu}-$ ñoz de la Fuente, 2010).

Dinamarca permite que la mujer acceda libremente a las técnicas de reproducción asistida, pero no contempla la fecundación postmortem como una opción (Muñoz de la Fuente, 2010).

\section{Situación Internacional}

En Colombia no existe una legislación específica para tal caso, por lo que se procederá a la fecundación postmortem si el semen estaba ya extraído antes de la muerte del hombre, con lo cual se supone había un consentimiento previo; en caso contrario la legislación no 
está preparada para acatar el problema, por lo que posiblemente el comité de ética al que se solicite el permiso oportuno, en cada hospital, tendrá que estudiar detenidamente el caso y cerciorarse de que no se buscan beneficios económicos en cuanto a patrimonios, etc. (Barbosa, Cardenas, Patiño, Noguera, Brigard, Echeverri, et al., 2015).

Argentina, Mexico y Chile tampoco cuentan con leyes propias en esta materia. Recientemente se describió en Argentina un caso en el que una pareja comenzó el proceso para someterse a tratamiento de fertilidad, extrayéndose en aquel momento el material genético al varón para su posterior uso, el hombre firmó el consentimiento para realizar el proceso y utilizar el material con fines reproductivos, al poco tiempo el varón falleció a causa de un tumor y la señora pidió los permisos oportunos para poder disponer del material, dándose por aprobada la petición. Hay que tener en cuenta que no se había fecundado el óvulo, sólo se disponía del material genético del marido, y aun así aceptaron los permisos (Pérez, 2012).

Israel por el contrario, lo contempla como una opción para las viudas, no siendo necesario un consentimiento previo por parte del marido (Gómez Jerez, 2006).

En Estados Unidos es de libre elección realizar cualquier tipo de tratamiento de terapia de reproducción humana asistida y no hay ninguna legislación que lo regule espe- cíficamente, por lo que es de libre acceso la fecundación postmortem, aunque algunos estados pueden requerir el consentimiento previo del fallecido (Muñoz de la Fuente, 2010).

Canadá no permite el uso de material genético sin el consentimiento del donante fallecido, pero sí permite la transferencia de embriones creados mientras el hombre estaba en vida, ya que supone, es algo que tenían en un plan común como matrimonio (Muñoz de la Fuente, 2010). A continuación, se expone una tabla (Tabla 1) con la legislación vigente respecto a la fecundación post-mortem en algunos países:

TABLA 1

\begin{tabular}{|c|c|c|c|c|}
\hline & $\begin{array}{l}\text { Prohibido por } \\
\text { legislación }\end{array}$ & $\begin{array}{l}\text { Consentimiento } \\
\text { informado por } \\
\text { escrito }\end{array}$ & $\begin{array}{c}\text { Sin } \\
\text { consentimiento } \\
\text { informado } \\
\text { escrito }\end{array}$ & $\begin{array}{l}\text { No hay guías ni } \\
\text { legislación } \\
\text { específicas }\end{array}$ \\
\hline Alemania & $\mathrm{X}$ & & & \\
\hline Australia & & & $\mathrm{X}$ & $\mathrm{X}$ \\
\hline Bélgica & & & $\mathrm{X}$ & $\mathrm{X}$ \\
\hline Canadá & $\mathrm{X}$ & & & \\
\hline Chipre & & & & $\mathrm{X}$ \\
\hline Colombia & & & & $\mathrm{X}$ \\
\hline Dinamarca & $\mathrm{X}$ & & & \\
\hline EEUU & & & $\mathrm{X}$ & $\mathrm{X}$ \\
\hline Estonia & $\mathrm{X}$ & & & \\
\hline Eslovaquia & & & & $\mathrm{X}$ \\
\hline Eslovenia & $\mathrm{X}$ & & & \\
\hline España & & $\mathrm{X}$ & & \\
\hline Francia & $\mathrm{X}$ & & & \\
\hline Hungría & $\mathrm{X}$ & & & \\
\hline Holanda & $\mathrm{X}$ & & & \\
\hline Irlanda & & & & $\mathrm{X}$ \\
\hline Israel & & & $\mathrm{X}$ & \\
\hline Italia & & & & $\mathrm{X}$ \\
\hline Japón & & & & $\mathrm{X}$ \\
\hline Letonia & & & & $\mathrm{X}$ \\
\hline Lituania & & & & $\mathrm{X}$ \\
\hline Malta & & & & $X$ \\
\hline Noruega & $\mathrm{X}$ & & & \\
\hline Polonia & & & & $X$ \\
\hline Reino Unido & & $\mathrm{X}$ & & \\
\hline República Checa & & & $\mathrm{X}$ & \\
\hline Suecia & $\mathrm{X}$ & & & \\
\hline
\end{tabular}

Fuente: Modificada de Barbosa, 2015 


\section{DISCUSIÓN}

Aún son muchos los países que no tienen una legislación específica referente a la FIV postmortem, de hecho el número de países sin legislación supera con creces al de países con una ley actual y acorde a la situación, siendo pocos los países que permiten la realización de ésta técnica, incluso con la autorización expresa del padre.

Alemania, Canadá, Dinamarca, Estonia, Eslovenia, Francia, Hungría, Holanda, Noruega y Suecia prohíben la extracción de semen peri mortem (ESP), mientras que Reino Unido y España lo limitan al previo consentimiento por escrito del varón (situaciones especiales). En Estonia puede usarse el semen extraído antes de 1 mes siempre y cuando la pareja estuviera incluida en un programa de reproducción asistida. Australia, República Checa, EEUU y Bélgica no necesitan el consentimiento por escrito. En Israel sólo es posible por orden de una Corte (no hay legislación especifica). No tienen guías ni legislación definida Australia, Bélgica, Chipre, Colombia, EEUU, Eslovaquia, Irlanda, Italia, Japón, Letonia, Lituania, Malta, Polonia.

\section{CONCLUSIONES}

A modo de conclusión se puede afirmar que este tema genera bastante polémica ya que apela a cuestiones legales y éticas. Existe gran controversia en cuanto a la legislación en distintos países, ante las grandes diferencias en el tratamiento de una misma cuestión. Mientras algunos países abogan por la prohibición y la penalización, y otros muestran total permisividad para realizar la fecundación postmortem.

Ante esta realidad, se plantea el escenario en el que mujeres que deseen cumplir su deseo de ser madres intenten acogerse a la legislación de un país vecino.
Es prospectiva la elaboración de normas legales en colaboración con juristas, profesionales de la salud e investigadores sociales, que contemplen las distintas posibilidades que pueden darse ante la demanda de una FIV postmortem. Además sería deseable que existiese un consenso internacional mediante organismos supragubernamentales que velases por los derechos de todos los individuos implicados en la realización de esta técnica.

\section{BIBLIOGRAFÍA}

- Barbosa, S., Cardenas, D., Patiño, J.F., Noguera, E., Brigard, A.M.D., Echeverri, S. et al (2015). Extracción de semen post mortem: aspectos éticos y legales. Descripción de caso y estudio en Colombia. Revista Colombiana de Boiética, 10(1), 170-181. Recuperado de http://doi.org/10.18270/rcb.v10i1.690

- Constitución Española. (29 de Diciembre de 1978). Boletín Oficial del Estado. Recuperado de https://www.boe. es/diario boe/txt.php?id=BOE-A-1978-31229

- Escudero Velando, L.E. (2012). Estimulación ovárica en reproducción asistida. Revista Peruana de Ginecologia y Obstetricia, 58 (3), 191-200. Recuperado de https://apps.webofknowledge.com/full_record. $\underline{\text { do?product }=U A \& \text { search } \text { mode }=\text { GeneralSearch \&qid }=5}$ $\underline{\& S I D=N 19 m I t N i l I A D j o 33 E z 5 \& p a g e=1 \& d o c=18}$

- Gobierno de España. (24 de Diciembre de 1988). Ley 35/1988, sobre Técnicas de Reproducción Asistida. Boletín Oficial del Estado. Recuperado de https://www.boe. es/buscar/doc.php?id=BOE-A-1988-27108

- Gobierno de España. (27 de Mayo de 2006). Ley 14, sobre técnicas de reproducción humana asistida. Boletín Oficial del Estado. Recuperado de https:// www.boe.es/buscar/act.php?id=BOE-A-2006$9292 \& \mathrm{~b}=12 \& \operatorname{tn}=1 \& \mathrm{p}=20150714 \# \mathrm{a} 9$

- Gómez Jerez, P.L. (2006). Fecundación Post-Mortem. Caracas:Tecnicaspa. Recuperado de http://www.monografias.com/trabajos91/fecundacion-post-mortem/ fecundacion-post-mortem.shtml\#tecnicaspa

- Muñoz de la Fuente, Y. (2010). La fecundación post mor- 


\section{Cultura de las Cuidados}

tem. Memoria del Master en Derecho de Familia, Infancia y Adopción. Barcelona.

- Pastrana Sánchez, M.A. (2014). Algunos problemas derivados de la aplicación de la ley española de técnicas de reproducción asistida. Revista Via Iuris, 16,177186. Recuperado de http://www.redalyc.org/articulo. $\underline{\text { oa? } \mathrm{id}=273933373010}$

- Pérez, A.G. (2012). Fertilización post mortem: un supuesto especial entre las técnicas de reproducción humana asistida. Trabajo presentado en Congreso de Derecho Privado para estudiantes y jóvenes graduados Reflexiones sobre la reforma del Código Civil. Universidad de Buenos Aires. Buenos Aires. Argentina.

- Pérez Gallardo, L.B. ( 2007). Inseminación artificial y transferencia de preembriones post mortem: procreación y nacimiento más allá de los límites de la existencia humana. IUS. Revista del Instituto de Ciencias Jurídicas de Puebla A.C, 139-163. Recuperado de http://www.redalyc.org/articulo.oa? $\mathrm{id}=293222932009$

- Rodríguez Guitián, A.M. (2015). La reproducción artificial post mortem en España: estudio ante un nuevo dilema jurídico. Revista Boliviana de Derecho, 20, 292-323. Recuperado de http://idibe.org/wp-content/ uploads/2013/09/121.pdf

- Tash, J., Applegarth, L.D., \& Kerr S.Ml. (2003). Postmortem sperm retrieval: The effect of instituting guidelines. The Journal of Urology, 170 (5), 1922-1925. Recuperado de http://www.jurology.com/article/S00225347(05)62965-5/fulltext

- Vega, M., Vega, J., \& Martinez Baza P. (2004). Regulación de la Reproducción Asistida en el ámbito europeo. Valladolid. Recuperado de http://www.bioeticaweb.com/ $\underline{\text { regulacion-de-la-reproduccion-asistida-en-el-ambito- }}$ europeo/

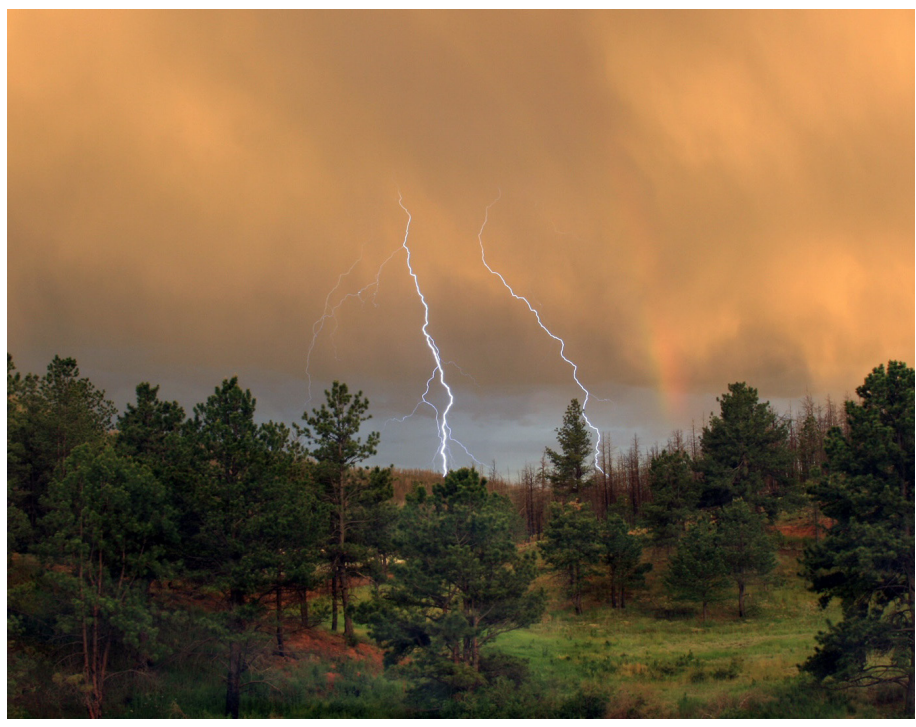

